\title{
Comparison between Two Algorithms for Multi-Depot Vehicle Routing Problem with Inventory Transfer between Depots in a Three-Echelon Supply Chain
}

\author{
Farzaneh Amiri Fard \\ Graduated Student \\ Department of Industrial Engineering, K.N. Toosi \\ University of Technology, Tehran, Iran
}

\author{
Mostafa Setak \\ Assistant Professor \\ Department of Industrial Engineering, K.N. Toosi \\ University of Technology, Tehran, Iran
}

\begin{abstract}
Coordination in supply chain plays an important role on the successful performance of all parts of supply chain. This paper studies an integrated distribution system in a three-echelon supply chain including a single plant, multiple distribution centers and a set of retailers with deterministic demands. Possibility of transferring goods between depots is taken into account. To solve the problem, first we formulate a mixed integer programming model to the overall system. Since solving mixed integer programming problems with optimization solvers is memory intensive and insufficient physical memory is one of the most common problems when running large size of these problems, we propose two approaches to solve the model and compare them. First approach is a constructive two-phase heuristic: The purpose of the first phase is to assign retailers to distribution centers and determine the source of inventory replenishment for each depot. After assigning retailers to the depots, sequence of routes for each depot is determined with a Simulated Annealing algorithm. Second approach is a Tabu search algorithm with different neighborhood structures that solve the model integrally, not in two phases. Computational results indicate the effectiveness of two proposed algorithms but when the integrated algorithm is used, better results achieved.
\end{abstract}

\section{Keywords}

Multi-depot vehicle routing problem, inventory transfer, mixed integer programming, heuristic algorithm, Simulated Annealing, Tabu search.

\section{INTRODUCTION}

In recent years due to increased competition, supply Chain Management (SCM) has received more attention for specialists and researchers. Simchi-levi et al. (2003) stated that " SCM is a set of approaches utilized to efficiently integrate suppliers, manufacturers, warehouses and stores, so that merchandise is produced and distributed at the right quantities, to the right location, and at the right time, in order to minimize system-wide costs while satisfying service level requirements". Since all parts of a supply chain are tightly connected, coordination of these parts results in minimizing costs of system and better response to their customers need. Coordination in supply chain may occur between these functions: production, distribution and inventory control. In this research we focus on coordination of inventory and distribution in a three-echelon supply chain. In fact we combine two type of problem: Vehicle Routing Problem (VRP) and inventory control that is called Inventory Routing Problem (IRP).

The first paper in the VRP context was presented by Dantzig et al. (1954). They studied a Traveling Salesman Problem (TSP) that is a specific version of VRP with one vehicle. Then Clarke and Wright (1964) studied a TSP with more than one vehicle. Golden et al. (1984) were the first ones who used the term inventory/routing problem for vehicle routing problems that have an inventory component. Campbell et al. (1997) explained that "IRP is concerned with the repeated distribution of a single product, from a single facility, to a set of customers over a given planning period". This is the base definition for an IRP but all inventory routing problems don't match with this definition in all aspects.

Although most IRP papers have two layers but there are a few papers with more than two layers. Shen and Qi (2007) consider the design of a three-tiered supply chain system consisting of one or more suppliers, distribution centers (DCs) and customers where the decision maker needs to decide the number and locations of the distribution centers. An integrated inventory and routing problem in a three-echelon logistics system has been studied by Zhao et al. (2008) . They proposed the fixed partition and power-of-two (FP-POT) policy and a variable large neighborhood search (VLNS) algorithm for solving their problem.

Multi-depot Vehicle Routing Problem (MDVRP) is a generalization of the VRP. A three-phase heuristic/algorithmic approach for the multi-depot routing problem with time windows and heterogeneous vehicles is presented by Dondo and Cerda (2007).

None of these papers considered the Possibility of transfer goods between distribution centers (depots) whereas adding this feature may reduce total cost of the problem. To our knowledge in recent years there are only two papers which involve this possibility in their formulation. Crevier et al. (2007) addressed an extension of the multi-depot vehicle routing problem in which vehicles may be replenished at intermediate depots along 
their route. Also Shen and Honda (2009) formulated lateral transfers of both vehicles and inventory in a three-echelon supply chain system including a single plant, multiple distribution centers and multiple retailers. They consider direct shipping with full-truck load for distribution from plant to depots, but if the products are perishable and we use the fulltruck load policy, we face additional costs for remaining inventory and this method shouldn't be used. So we didn't use the full-truck load policy in our formulation and let the quantity transported from plant to depots be variable.

In this paper we consider not only optimization of a multi-depot vehicle routing problem, but also the upper layer of supply chain that is transferring goods from a plant to depots and possibility of transferring goods between depots is considered.

In section 2 we first formulate a mixed integer programming model to the problem. Section 3 describes the first heuristic approach to solve the problem. In section 4 we propose the second approach that is a Tabu Search algorithm. Section 5 reports the numerical results and compare two proposed algorithm. Finally, section 6 gives some conclusions and further research issues.

\section{MODEL FORMULATION}

Consider a three-echelon supply chain where in the first level, there is a plant that has unlimited capacity to produce one type of a product. In the second level there are several distribution centers as the possibility of transferring goods between them is considered. In the Third-level there are a set of geographically scattered customers. Location of all facilities has been determined. The problem is to determine the plan of distributing product \{allocate customers to distribution centers, determine the vehicles routing and the resource of inventory for distribution centers (plant or other depots) $\}$ such as the transportation costs is minimized.

In a single period planning horizon, customers demand is known for the plant. Initial inventory for customers and distribution centers is to be zero. The lack of inventory is not allowed. Each customer will be assigned to one distribution center. Also each customer demand can be satisfied only by a vehicle without split delivery. Table 1 explains the notation used for the mixed integer programming model presented below.

Table 1 : Notations used in the model

\begin{tabular}{|l|}
\hline \multicolumn{1}{|c|}{ Parameters } \\
\hline $\mathrm{D}=$ set of depots \\
\hline $\mathrm{R}=$ set of customers \\
\hline$v_{d}=$ fleet of vehicles of depot d \\
\hline$F_{0}=$ Fixed cost incurred when using a vehicle belonging to the plant \\
\hline$F_{d}=$ Fixed cost incurred when using a vehicle belonging to the $\mathrm{d} \in D$ \\
\hline$c_{d}=$ Traveling cost from plant to depot d \\
\hline$c_{i, j}=$ Traveling cost from location $\mathrm{i}$ to location $\mathrm{j}$ \\
\hline$V_{d}=$ Number of vehicles contained in set $v_{d}$ \\
\hline$Q_{0}=$ Capacity for vehicles stationed at plant \\
\hline$Q_{d}=$ Capacity for vehicles stationed at each depot \\
\hline$r_{i}=$ Product demand of retailer i \\
\hline \multicolumn{1}{|c|}{ Decision variables } \\
\hline$x_{d}=$ Number of trips replenishing goods from the plant to depot d \\
\hline$p_{d}=$ The amount of goods transferred from the plant to $\mathrm{d} \in D$ \\
\hline
\end{tabular}

$t_{d, d^{\prime}, v_{d}}=$ Number of trips of transferring goods from $\mathrm{d} \in D$ to $d^{\prime} \in D$ $s_{d, d^{\prime}}=$ The amount of goods transferred from $\mathrm{d} \in D$ to $d^{\prime} \in D$

$w_{i, j}=$ quantity transported through the directed $\operatorname{arc}(\mathrm{i}, \mathrm{j})$

$y_{i, j}=\{1$; if the directed $\operatorname{arc}(\mathrm{i}, \mathrm{j})$ is visited by vehicles

$y_{i, j}=\left\{\begin{array}{c}1 ; \text { otherwise } \\ 0 ; \text { the }\end{array}\right.$

$z_{i, d}=\{1 ;$ if retailer $\mathrm{i}$ is assigned to $\mathrm{d} \in \mathrm{D}$

$\mathrm{Z} \& \mathrm{y}$ are binary, $\mathrm{x} \& \mathrm{t}$ are positive integer and other variables are positive.

Equation (1) is the objective function which minimizes these terms respectively: fixed and variable costs of delivering goods from plant to the depots, fixed and variable cost of using vehicles belonged to each depot for transferring goods between depots, traveling cost between customers, fixed and variable cost of using vehicles belonged to each depot for distributing goods from depots to customers.

Equation (2) is capacity constraint of vehicles belonging to the plant. Constraint (3) requires that the number of trips regarding transferring goods between depots and between a depot and customers cannot be greater than the total number of vehicles belonged to the original depot. Constraint (4) means that the total amount of goods transferred between two depots cannot be greater than the number of respected trips for transferring goods times the vehicle capacity. Constraint (5) is the inventory balance constraint of each depot. Constraint (6) restricts that each retailer must be assigned to a distribution center exactly once.

Constraints (7) and (8) ensure that if retailers $i$ and $j$ are not assigned to the same DC, there will be no vehicles to serve arc $(i, j)$. Constraint (9) ensures that each retailer will be visited only by one vehicle exactly once. Constraint (10) says that the number of vehicles leaving from a customer is equal to the number of its arrival vehicles. Constraints (11a) and (11b) model the vehicle capacity and logical relationship between $\mathrm{w}$ and $\mathrm{y}$. Constraint (12) is the product flow conservation equations, assuring the flow balance at each customer, and with constraints (11a) and (11b) eliminating all sub-tours. 


$$
\begin{aligned}
& \operatorname{Min} Z=\sum_{d \in D}\left(F_{0}+2 c_{d}\right) * x_{d}+\sum_{d \in D} \sum_{d^{\prime} \in D}\left(F_{d}+2 c_{d, d^{\prime}}\right) * t_{d, d^{\prime}, v_{d}} \\
& d \neq d^{\prime} \\
& +\sum_{j \in R} \sum_{i \in R} c_{i, j} * y_{i, j}+\sum_{d \in D} \sum_{i \in R} c_{d, i} *\left(F_{d} * y_{d, i}+y_{i, d}\right) \\
& i \neq j \\
& p_{d} \leq Q_{0} * x_{0, d} \quad \forall d \in D \\
& \sum_{d^{\prime} \in D / d} t_{d, d^{\prime}, v_{d}}+\sum_{i \in R} y_{d, i} \leq V_{d} \quad \forall d \in D \\
& s_{d, d^{\prime}} \leq Q_{d} \times t_{d, d^{\prime}, v_{d}} \quad \forall d, d^{\prime} \in D, d \neq d^{\prime} \\
& p_{d}+\sum_{d^{\prime} \in D / d} s_{d^{\prime}, d}-\sum_{d^{\prime} \in D / d} s_{d, d^{\prime}}=\sum_{i \in R} r_{i} \times z_{i, d}, \forall d \in D \\
& \sum_{d \in D} z_{i, d}=1 \quad \forall i \in R \\
& y_{i, d}+y_{d, i} \leq 2 z_{i, d} \quad \forall i \in R, d \in D \\
& y_{i, j}+y_{j, i} \leq 2-\left(z_{i, d}+\sum_{d^{\prime} \in D} z_{j, d^{\prime}}\right) \forall i, j \in R, i<j, d \in D \\
& d \neq d^{\prime} \\
& \sum_{i \in R \cup D} y_{i, j}=1 \quad \forall j \in R \\
& \mathrm{i} \neq \mathrm{j} \\
& \sum_{j \in R \cup D} y_{j, i}=\sum_{j \in R \cup D} y_{i, j} \quad \forall i \in R \\
& i \neq j \quad i \neq j \\
& w_{i, j} \leq \operatorname{MAX}\left\{Q_{d}\right\} \times y_{i, j} \quad \forall i, j \in R \\
& \text { d } \\
& w_{d, i} \leq Q_{d} \times y_{d, i} \quad \forall i \in R, \forall d \in D \\
& \sum_{j \in R \cup D} w_{j, i}-\sum_{j \in R} w_{i, j}=r_{i} \quad \forall i \in R \\
& i \neq j \quad i \neq j
\end{aligned}
$$




\section{FIRST APPROACH: A HEURISTIC ALGORITHM}

Since the vehicle routing problem is NP-hard, there are numerous variables in the model and it is difficult to be solved optimally in a reasonable time even for medium sizes. So we propose a constructive two-phase heuristic to obtain good and near optimum solutions. The purpose of the first phase is to assign retailers to distribution centers and determine the source of inventory replenishment for each depot so as the total cost is minimized. After assigning retailers to the distribution centers, the main problem is decomposed into several single depot vehicle routing problems. In the next phase, with a simulated annealing meta-heuristic sequence of routes for each depot is determined.

\subsection{Phase I: Assigning}

In this phase we use the proposed mixed integer model with some modifications for the up two level of supply chain. In this model we do not consider the traveling cost between customers and assign customers to the depots just in basis of nearness to the depots. The model is showed below:

$n_{d}$ : Number of tours originated from depot $d$.

Other notations are the same as the notations showed in table 1.

$$
\begin{array}{r}
\operatorname{Min} Z=\sum_{d \in D}\left(F_{0}+2 c_{d}\right) * x_{d}+\sum_{d \in D} \sum_{d^{\prime} \in D}\left(F_{d}+2 *\right. \\
\left.c_{d, d^{\prime}}\right) * t_{d, d^{\prime}, v_{d}}
\end{array}
$$

$$
\begin{aligned}
& d \neq d^{\prime} \\
& +\sum_{d \in D} F_{d} * n_{d}+\sum_{d \in D} \sum_{i \in R} c_{d, i} * z_{i, d} \\
& p_{d} \leq Q_{0} * x_{d} \quad \forall d \in D \\
& \sum_{d^{\prime} \in D / d} s_{d, d^{\prime}}+\sum_{i \in R} r_{i} * z_{i, d} \leq V_{d} * Q_{d} \quad \forall d \in D \\
& p_{d}+\sum_{d^{\prime} \in D / d}^{s_{d, d^{\prime}} \leq Q_{d} \times t_{d, d^{\prime}, v_{d}}} s_{d^{\prime}, d}-\sum_{d^{\prime} \in D / d}^{\forall d, d^{\prime} \in D, d \neq d^{\prime}} s_{d, d^{\prime}}=\sum_{i \in R} r_{i} \times z_{i, d} \quad \forall d \in D \\
& \begin{array}{cl}
\sum_{d \in D} z_{i, d}=1 & \forall i \in R \\
\sum_{i \in R} r_{i} * z_{i, d} / Q_{d} \leq n_{d} & \forall d \in D
\end{array}
\end{aligned}
$$

\subsection{Phase II: Simulated Annealing}

After assigning customers to the depots, then a simulated annealing algorithm for solving the single depot VRP is presented. The algorithm of simulated annealing which can be regarded as a variant of local search was first introduced by Metropolis et al. (1953) and then used to optimization problems by Kirkpatrick et al. (1983).
- $\quad$ Steps of SA

Let us summarize the steps of the Simulated Annealing. 1. Initialization:

1.a. Initialize the first solution(s), calculate its cost,

1.b. Initialize SA parameters and termination limit.

2. Start a loop, while we are above the limit, repeat:

2.a. with the "neighbor" function, find new solution

2.b. and evaluate its cost.

2.c. If this cost is better than the one before, save it as current (s) and current cost.

2.d. If it's not, with a possibility function defined in step $b$ move to new solution.

2.e. Finally decrease temperature.

3. As we are under the limit, we return the solution (s).

In proposed algorithm a solution is represented with 2 strings: 1 ) a random sequence of $\mathrm{V}$ (number of vehicles belonged to the specified depot) in a $1 * \mathrm{~V}$ array, 2) a random permutation of $\mathrm{R}$ customers in a $1 * \mathrm{R}$ array, e.g.: with 5 customers $(\mathrm{a}, \mathrm{b}, \mathrm{c}, \mathrm{d}, \mathrm{e})$ assigned to the depot and 2 vehicles $(1,2)$ the solution is showed as:

String 1: 2, 1, 1, 2, 1

String 2: b, d, c, e, a

Two routes will be: for vehicle $1: \mathrm{d} \rightarrow \mathrm{c} \rightarrow \mathrm{a}$

For vehicle $2: \mathrm{b} \rightarrow \mathrm{e}$

That both routes initialize and end to the depot. These routes may be impossible due to vehicle capacity constraint but we model a punishment cost for this case in the objective function which omit all invalid tours.

The neighborhood structure in the neighborhood function is defined as below:

- Swap: change the place of 2 customers in the route strings.

- Reversion: change the place of customers $i$ and $j$ and reverse the order of customers between them.

- Insertion: insert customer j after i.

- Another neighborhood may be: change the order of vehicles in string 1 .

One or combination of more than one of these neighborhood structures can be chosen.

\section{SECOND APPROACH: INTEGRATED TABU SEARCH META-HEURISTIC}

Second approach is an integrated Tabu Search algorithm with different neighborhood structures that solve the model integrally, not in two stages. Tabu search is an iterative procedure for building new solutions with neighborhood structures. It uses a tabu list in order to avoid being caught in a local optimum. Tabu list consists of number of recently visited solutions. This optimization Meta-heuristic was initially proposed by Glover (1977).

Steps of the algorithm is as follows.

1. Choose an initial solution to start the process. This solution is the present best solution.

2. Start a loop, while we are above the limit, repeat:

2.a.Scan the entire neighborhood of the current solution that are not in tabu list in search of the best feasible solution (one with the most desirable value of objective function). 
3. If no such solution can be found, the current solution is the local optimum, and the method stops. Otherwise, replace the best solution with the new one, and send this solution to the tabu list in order to avoid cycling, then go to step 2.

First we create a random solution. The solution is represented with 3 strings:

- $\quad \mathrm{T}(\mathrm{v})$ : a random permutation of $\mathrm{R}$ customers in a $\mathrm{V}^{*} 1$ cell array $(\mathrm{V}=$ total number of vehicles belonged to the all depots). Each cell belongs to a vehicle and total demands of customers assigned to a vehicle shouldn't be greater than the vehicle capacity. These cells determine the routes between customers and also assign customers to the depots.

- $\quad \mathrm{I}(\mathrm{v}):$ a $1 * \mathrm{~V}$ array which assign the amount of goods transferred between depots by vehicle $\mathrm{v}$.

- $\quad \mathrm{A}(\mathrm{v}):$ a $1 * \mathrm{~V}$ array which determines transferring goods between depots, if a cell(vehicle) in $\mathrm{T}(\mathrm{v})$ is empty, it choose a depot randomly, if this depot is not the depot that this vehicle belonged to and if $\mathrm{I}(\mathrm{v})$ is not equal to zero, it means the vehicle $\mathrm{v}$ transfers amount of goods ( equal to I(v)) between its original depot to the depot specified in $\mathrm{A}(\mathrm{v})$.

After determining the amount of $\mathrm{T}(\mathrm{v}), \mathrm{A}(\mathrm{v})$ and $\mathrm{I}(\mathrm{v})$, we can determine the amount of goods should be delivered from the plant to each depot with inventory balance constraint.
The neighborhood structures in the neighborhood function are: swap two customers in T, swap two vehicles in A, Assign a tour to best depot, Assign a customer to best tour ( choose a customer randomly and insert it in the best place in all tours), Assign the amount of transferring good (I) to the best depot, Break the amount of transferring good ( $I=0)$, change the amount of $I(v)$.

\section{COMPARISON OF RESULTS}

The mixed integer programming model and the first phase of the heuristic algorithm is programmed with GAMS 23.5 and the SA and TS meta-heuristics are programmed with MATLAB R2008a. Computational testing is run on a personal computer with $2.0 \mathrm{GHZ}$ processor and $2 \mathrm{~GB}$ of RAM.

The two proposed algorithm are implemented on a set of randomly generated examples, and then they are compared with optimal solutions obtained from the mixed integer programming model in medium sizes. The example contains one plant, three depots and up to 20 customers. Table 2 shows the results.

- Utilization of the vehicles is obtained from dividing total demand to the number of vehicles used multiplied to vehicle capacity.

- Number of customers is considered up to 20 customers, because with increasing number of customers, run time increases rapidly So that solving the problem through the optimization software does not seem logical.

TABLE 2: Comparison between MIP, 2-phase Heuristic and Tabu Search

\begin{tabular}{|c|c|c|c|c|c|c|c|c|}
\hline \multirow[b]{3}{*}{$\begin{array}{c}\text { Problem } \\
\text { no. }\end{array}$} & \multirow[b]{3}{*}{$\begin{array}{l}\text { Number of } \\
\text { customers }\end{array}$} & \multicolumn{7}{|c|}{ Results } \\
\hline & & \multicolumn{3}{|c|}{ Optimum (MIP) } & \multicolumn{4}{|c|}{ First Approach ( 2-phase Heuristic) } \\
\hline & & $\begin{array}{l}\text { Total cost } \\
\text { (currency) }\end{array}$ & $\begin{array}{c}\text { Processing } \\
\text { time(second) }\end{array}$ & $\begin{array}{c}\text { Utilization of } \\
\text { the vehicles } \\
\text { of depots }(\%)\end{array}$ & $\begin{array}{l}\text { Total cost } \\
\text { (currency) }\end{array}$ & $\begin{array}{c}\text { Processing } \\
\text { time(second) }\end{array}$ & $\begin{array}{c}\text { Utilization of } \\
\text { the vehicles } \\
\text { of depots }(\%)\end{array}$ & $\begin{array}{c}\text { Gap of } \\
\text { total cost } \\
(\%) \\
\end{array}$ \\
\hline $5 r$ & 5 & 3129 & 1 & 95.5 & 3129 & 3.1 & 95.5 & 0.0 \\
\hline $10 \mathrm{r}$ & 10 & 4973 & 12.9 & 84.5 & 5086 & 3.3 & 87.2 & 2.22 \\
\hline $11 \mathrm{r}$ & 11 & 5748 & 51.3 & 78.3 & 5766 & 52.9 & 94.7 & 0.31 \\
\hline $12 \mathrm{r}$ & 12 & 5750 & 16.6 & 82.6 & 6396 & 77.1 & 87.1 & 10.1 \\
\hline $13 r$ & 13 & 6073 & 39.9 & 83.5 & 6295 & 54.1 & 83.5 & 3.5 \\
\hline $14 r$ & 14 & 6477 & 578.1 & 91.3 & 6477 & 54.9 & 91.3 & 0.0 \\
\hline $15 r$ & 15 & 7226 & 1977.7 & 81 & 7567 & 80 & 95.5 & 4.5 \\
\hline $16 r$ & 16 & 7742 & 3495.6 & 90.8 & 7977 & 77.9 & 82 & 2.9 \\
\hline $17 \mathrm{r}$ & 17 & 7958 & 2621.1 & 92.2 & 8698 & 104.3 & 83.3 & 8.5 \\
\hline $18 \mathrm{r}$ & 18 & 8004 & 1224.5 & 86.2 & 8004 & 79.2 & 86.2 & 0.0 \\
\hline $19 \mathrm{r}$ & 19 & 9065 & 17540.7 & 81.2 & 9461 & 91.2 & 94.2 & 4.2 \\
\hline \multirow[t]{14}{*}{$* 20 \mathrm{r}$} & 20 & 9108 & 5793 & 92 & 8964 & 87.4 & 93.1 & -1.6 \\
\hline & & & & Problem no. & \multicolumn{4}{|c|}{ Second Approach ( Tabu Search) } \\
\hline & & & & $5 \mathrm{r}$ & 3129 & 46 & 95.5 & 0.0 \\
\hline & & & & $10 \mathrm{r}$ & 4973 & 75.6 & 84.5 & 0.0 \\
\hline & & & & $11 \mathrm{r}$ & 5748 & 190.2 & 78.3 & 0.0 \\
\hline & & & & $12 \mathrm{r}$ & 6027 & 137.3 & 87.8 & 4.6 \\
\hline & & & & $13 r$ & 6331 & 144.1 & 77.6 & 4.1 \\
\hline & & & & $14 \mathrm{r}$ & 6477 & 345 & 91.3 & 0.0 \\
\hline & & & & $15 r$ & 7226 & 352.5 & 81 & 0.0 \\
\hline & & & & $16 \mathrm{r}$ & 7742 & 404.8 & 90.8 & 0.0 \\
\hline & & & & $17 \mathrm{r}$ & 8093 & 490.1 & 81.7 & 1.7 \\
\hline & & & & $18 \mathrm{r}$ & 8004 & 516.7 & 86.2 & 0.0 \\
\hline & & & & $19 r$ & 9065 & 762 & 81.2 & 0.0 \\
\hline & & & & $* 20 \mathrm{r}$ & 8964 & 87.4 & 93.1 & -1.6 \\
\hline
\end{tabular}

* Best value in the case of out of memory 
Table 2 shows that there is small gap between optimal solutions and the solutions resulted from the first algorithm. But if the first phase of algorithm assigns customers to the depots optimally, the SA heuristic gives the optimal solution. In fact the gap between optimum solution and the heuristic is because of the first phase of the algorithm. When the integrated Tabu search algorithm is used, in most of times the gap between MIP and TS solutions is equal to zero.
Utilization of vehicles in all methods is more than 85 percent in average. In terms of processing time the two algorithms work better than optimality approach and in case of out of memory status that optimum solution cannot be obtained, these two approaches can achieve a solution much better from which obtained by MIP in a very short time. Computational results indicate the effectiveness of two proposed algorithms.

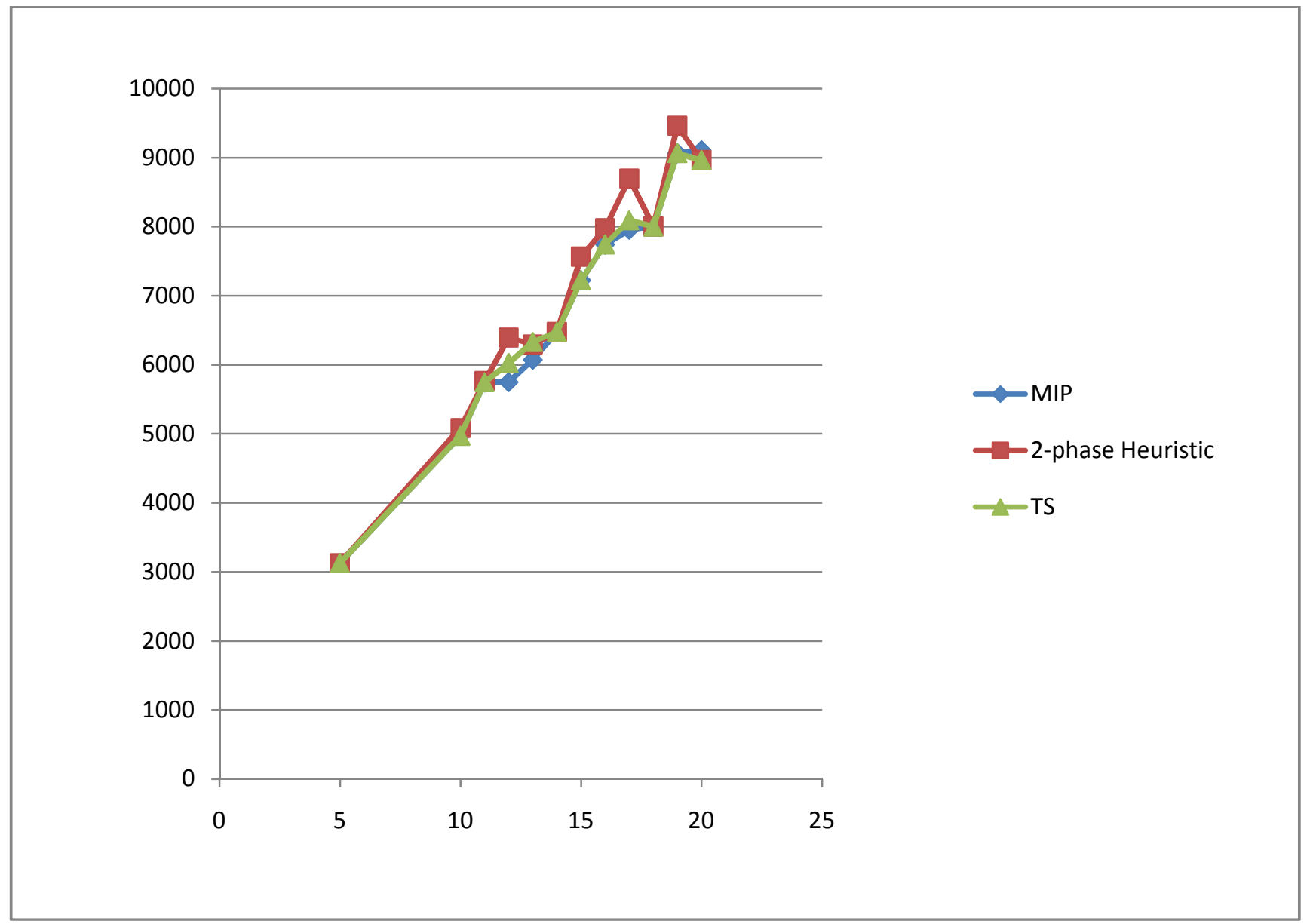

FIG1 : Comparison between costs of MIP, 2-phase Heuristic and Tabu Search

FIG 1 shows that the overlap between costs of TS and MIP solutions is more than between 2-phase heuristic and MIP solutions.

\section{CONCLUSION}

This paper presents a comparison between two Meta-heuristic solution approaches for multi-depot vehicle routing problem in a three-echelon supply chain with possibility of inventory transfer between depots. The Mixed Integer Programming model written in this paper specifies optimal allocation of customers to the depots and optimal paths between depots and customers simultaneously.

Since the vehicle routing problem is NP-hard, it is difficult to be solved optimally in a reasonable time even for medium sizes. So we propose a constructive two-phase heuristic and a Tabu Search Meta-heuristic to obtain good and near optimum solutions and then compare them to determine which is more efficient. Computational results show that integrated methods such as Tabu Search algorithm used in the second approach, are more efficient than heuristics based on a decomposition approach.

As described in introduction there are a few papers with more than two layers, so further studies in this area seems necessary. Developing the model to cases with multi-period, multi-product, probabilistic demand and considering cost of inventory can be directions for future research. 


\section{REFERENCES}

[1] Simchi-Levi, D., Kaminsky, P., and Simchi-Levi, E. 2003. Designing and managing the supply chain: concepts, strategies and case studies. Irwin, McGraw- Hill.

[2] G. Dantzig, R. Fulkerson, and S. Johnson, "Solution of a large-scale travelling salesman problem", Operations Research, vol. 2, 1954, pp. 393-410.

[3] G. Clarke, and J. W. Wright, "Scheduling of vehicles from a depot to a number of delivery points", Operations Research, vol. 12, 1964, pp. 568-581.

[4] B. L. Golden, A. Assad, and R. Dahl, "Analysis of a large scale vehicle routing problem with an inventory component”, Large Scale Systems, vol. 7, 1984, pp. 181190.

[5] Campbell, A. M., Clarke, L., Kleywegt, A., and Savelsbergh, M. W. P. 1997. Inventory routing. Fleet management and logistics. Kluwer Academic Publishers.

[6] Z. Shen, and L. Qi, "Incorporating inventory and routing costs in strategic location models", European Journal of Operational Research, vol. 172, 2007, pp. 372-389.

[7] Q. Zhao, S. Chen, and C. Zang, "Model and algorithm for inventory/routing decision in a three-echelon logistics system", European Journal of Operational Research, vol. 191, 2008, pp. 623-635.
[8] R. G. Dondo, and J. Cerda, "A cluster-based optimization approach for the multi-depot heterogeneous fleet vehicle routing problem with time windows", European Journal of Operational Research, vol. 176, 2007, pp. 1478-1507.

[9] B. Crevier, J. F. Cordeau, and G. Laporte, "The multi-depot vehicle routing problem with inter-depot routes", European Journal of Operational Research, vol. 176, 2007, pp. 756773.

[10] S. Shen, and M. Honda, "Incorporating lateral transfers of vehicles and inventory into an integrated replenishment and routing plan for a three-echelon supply chain", Computers \& Industrial Engineering, vol. 56, 2009, pp. 754-775.

[11] N. Metropolis, A.W. Rosenbluth, M. N. Rosenbluth, A. H. Teller and E. Teller, "Equation of state calculating by fast computing machines", Journ. Of chem. Phys, vol. 21, 1953, pp. 1087-1091.

[12] S. Kirkpatrick, C.D. Gellat and M. P. Vecchi, "Optimization by simulated annealing", Science, vol. 220, 1983, pp. 671-680.

[13] F. Glover, "Heuristic for integer programming using surrogate constraints", Decision Sciences, vol.8, 1977, pp. 66-156. 